\title{
Rings of constants of linear derivations on Fermat rings
}

\author{
Marcelo Veloso \\ e-mail: veloso@ufsj.edu.br \\ Ivan Shestakov \\ e-mail: shestak@ime.usp.br
}

\begin{abstract}
In this paper we characterize all the linear $\mathbb{C}$-derivations of the Fermat ring. We show that the Fermat ring has linear $\mathbb{C}$-derivations with trivial ring of constants and construct some examples.
\end{abstract}

Keywords: Derivations, Fermat ring, ring of constants.

2010 AMS MSC: 13N15, 13A50, 16W25.

\section{Introduction}

The present paper deals with $\mathbb{C}$-derivations of the Fermat ring

$$
B_{n}^{m}=\frac{\mathbb{C}\left[X_{1}, \ldots, X_{n}\right]}{\left(X_{1}^{m_{1}}+\cdots+X_{n}^{m_{n}}\right)},
$$

where $\mathbb{C}\left[X_{1}, \ldots, X_{n}\right]$ is the polynomial ring in $n$ variables over the complex numbers $\mathbb{C}, n \geq 3, m=\left(m_{1}, \ldots, m_{n}\right), m_{i} \in \mathbb{Z}$ and $m_{i} \geq 2$ for $i=1, \ldots, n$.

It is well known the difficulty to describe the ring of constants of an arbitrary derivation (see $[1,3,4,5]$ ). It is also difficult to decide if the ring of constants of a derivation is trivial (see $[5,6,7])$. In this work we study the ring of constants of linear derivations of Fermat rings and its locally nilpotent derivations.

In [5], Andrzej Nowicki presents a description of all linear $\mathbb{C}$-derivations of the polynomial ring $\mathbb{C}\left[X_{1}, \ldots, X_{n}\right]$ which do not admit any nontrivial rational constant.

In a recent paper [1], P. Brumatti and M. Veloso show that for $m=(2, \ldots, 2)$ the ring $B_{n}^{m}$ has nonzero irreducible locally nilpotent derivations. Furthermore, whenever $m_{1}=\cdots=m_{n}$, They show that certain classes of derivations of $\mathbb{C}\left[X_{1}, \ldots, X_{n}\right]$ do not induce derivations of $B_{n}^{m}$ or are not locally nilpotent if they do.

In this work we obtain some similar results to [1], considering more general Fermat rings. We present a description of all the linear $\mathbb{C}$-derivations of $B_{n}^{m}$ 
when $m=\left(m_{1}, \ldots, m_{n}\right)$ and $m_{i} \geq 3$ (Theorem 5$)$ and $m=(2, \ldots, 2)$ (Theorem 6). We also provide examples of linear derivations of $B_{n}^{m}$ with trival ring constants.

The text is organized as follows: Section 1 gathers the basic definitions and notations. Further, we discuss several properties of the ring $B_{n}^{m}$ are discussed and a set of generators for $\operatorname{Der}\left(B_{n}^{m}\right)$ is presented. Section 2 is dedicated to the study of the linear derivations of the Fermat ring. The set of all locally nilpotent $\mathbb{C}$-derivations of $B_{n}^{m}$ is studied in Section 3. Finally, Sections 4 and 5 are devoted to the study of the rings of constants of linear derivations of Fermat rings.

\section{Preliminaries and Some Results}

In this paper the word "ring" means a commutative ring with unit and characteristic zero. Furthermore, we denote the group of units of a ring $R$ by $R^{*}$ and the polynomial ring in $n$ variables over $R$ by $R\left[X_{1}, \ldots, X_{n}\right]$. A "domain" is an integral domain.

An additive mapping $D: R \rightarrow R$ is said to be a derivation of $R$ if it satisfies the Leibniz rule: $D(a b)=a D(b)+D(a) b$, for all $a, b \in R$. If $A$ is a subring of $R$ and $D$ is a derivation of $R$ satisfying $D(A)=0$ is called $D$ an $A$-derivation. The set of all derivations of $R$ is denoted by $\operatorname{Der}(R)$, the set of all $A$-derivations of $R$ by $\operatorname{Der}_{A}(R)$ and by $\operatorname{ker}(D)$, the ring of constants of $D$, that is $\operatorname{ker}(D)=\{a \in R \mid D(r)=0\}$.

In this paper, the word "derivation" implicitly means a derivation which is $\mathbb{C}$-derivation and therefore we will use the notation $\operatorname{Der}\left(B_{n}^{m}\right)$ to denote $\operatorname{Der}_{\mathbb{C}}\left(B_{n}^{m}\right)$. The residue classes of variables $X, Y, Z, \ldots$ module an ideal are represented by $x, y, z$, respectively. The symbol $\mathbb{C}$ is reserved to indicate the field of complex numbers.

A derivation $D$ is locally nilpotent if for each $r \in R$ there is an integer $n \geq 0$ such that $D^{n}(r)=0$. We denote by $L N D(R)$ the set of all locally nilpotent derivations of $R$.

We say that a element $b \in R$ is a Darboux element of $D \in \operatorname{Der}(R)$ if $b \neq 0, b$ is not invertible in $R$ and $D(b)=\lambda b$ for some $\lambda \in R$. In other words, a nonzero element $b$ of $R$ is a Darboux element of $D$ if, and only if, the principal ideal $(b)=\{r b \mid r \in R\}$ is different from $R$ and it is invariant with respect to $D$, that is $D((b)) \subset(b)$. If $b$ is a Darboux element of $D$, then every $\lambda \in R$, such that $D(b)=\lambda b$, is said to be an eigenvalue of $b$. In particular, every element nonzero and noninvertible element belonging to the ring of constants, ker $D$, is a Darboux element of $D$. If $R$ is a domain and $D(b)=\lambda b$, then it is easy to see such the eigenvalue $\lambda$ is unique.

Lemma 1 Let $B_{n}^{m}$ where $m=\left(m_{1}, \ldots, m_{n}\right)$. Then, for each $f \in B_{n}^{m}$, there is a unique $F \in \mathbb{C}\left[X_{1}, \ldots, X_{n}\right]$ such that $\operatorname{deg}_{X_{n}}<m_{n}$ and $f=F\left(x_{1}, \ldots, x_{n}\right)$.

Proof. It follows directly from the Euclidean division algorithm by considering the polynomial $X_{1}^{m_{1}}+\cdots+X_{n}^{m_{n}}$ as a monic polynomial in $X_{n}$ with coefficients 
in $\mathbb{C}\left[X_{1}, \ldots, X_{n-1}\right]$.

Theorem 2 ([3, Theorem 4]) If $n \geq 5$ and $m_{i} \geq 2$ for all $1 \leq i \leq n$, then $B_{n}^{m}$ is a unique factorization domain.

We also can write $B_{n}^{m}=\mathbb{C}\left[x_{1}, \ldots, x_{n}\right]$, where $x_{1}^{m_{1}}+\cdots+x_{n}^{m_{n}}=0$. Here $x_{1}, x_{2}, \ldots, x_{n}$ are the images of $X_{1}, X_{2}, \ldots, X_{n}$ under the canonical epimorphism $\mathbb{C}\left[X_{1}, \ldots, X_{n}\right] \rightarrow B_{n}^{m}$. An element of form $a x_{1}^{m_{1}} \cdots x_{n-1}^{m_{n-1}}$ or $b x_{1}^{m_{1}} \cdots x_{n_{1}}^{m_{n-1}} x_{n}^{j}$, for $1 \leq j \leq m_{n}-1$, is called monomial. A nonzero element $f \in \mathbb{C}\left[x_{1}, \ldots, x_{n}\right]$ is said to be homogeneous element of degree $k$ if $f$ is of the form

$$
f=\sum_{i_{1}+\cdots+i_{n}=k} a_{\left(i_{1} \cdots i_{n}\right)} x_{1}^{i_{1}} \cdots x_{n}^{i_{n}}
$$

where $1 \leq i_{n} \leq m_{n}-1$ and $a_{\left(i_{1} \cdots i_{n}\right)} \in \mathbb{C}$ for all $\left(i_{1} \cdots i_{n}\right)$. We assume that the zero element is a homogeneous element of any degree. Furthermore, we denote by $\mathcal{V}_{k}$ the set of all homogeneous elements of degree $k$. Clearly $\mathcal{V}_{k}$ is a subspace of $B_{n}^{m}$.

\subsection{A set of generators for $\operatorname{Der}\left(B_{n}^{m}\right)$}

Now we will present a set of generators for the $B_{n}^{m}$-module $\operatorname{Der}\left(B_{n}^{m}\right)$.

First some notation will be established. Given $H \in S=\mathbb{C}\left[X_{1}, \ldots, X_{n}\right]$ and $1 \leq i \leq n$, the partial derivative $\frac{\partial(H)}{\partial X_{i}}$ is denoted by $H_{X_{i}}$. For all pairs $i, j \in\{1, \ldots, n\}$ with $i \neq j$, we define the derivation $D_{H_{i j}}=H_{X_{i}} \frac{\partial}{\partial X_{j}}-H_{X_{j}} \frac{\partial}{\partial X_{i}}$ on $S$. Observe that $D_{H_{i j}}(H)=0$.

Let $A=\frac{\mathbb{C}^{[n]}}{I}$ be a finitely generated $\mathbb{C}$-algebra. Consider the $\mathbb{C}^{[n]}$-submodule $\mathcal{D}_{I}=\left\{D \in \operatorname{Der}_{\mathbb{C}}\left(\mathbb{C}^{[n]}\right) \mid D(I) \subseteq I\right\}$ of the module $\operatorname{Der}_{\mathbb{C}}\left(\mathbb{C}^{[n]}\right)$. It is well known that the $\mathbb{C}^{[n]}$-homomorfism $\varphi: \mathcal{D}_{I} \rightarrow \operatorname{Der}_{\mathbb{C}}(A)$ given by $\varphi(D)(g+I)=D(g)+I$ induces a $\mathbb{C}^{[n]}$-isomorfism of $\frac{\mathcal{D}_{I}}{I D e r_{\mathbb{C}}\left(\mathbb{C}^{[n]}\right)}$ in $\operatorname{Der}_{\mathbb{C}}(A)$.

The Theorem 3 will be needed, its proof can be found in [2, Proposition 1].

Theorem 3 Let $F \in \mathbb{C}^{[n]}=\mathbb{C}\left[X_{1}, \ldots, X_{n}\right](n \geq 2)$ be such that $\left\{F_{X_{1}}, \ldots, F_{X_{n}}\right\}$ is a regular sequence in $S$. If there exists a derivation $\partial$ on $S$ such that $\partial(F)=$ $\alpha F$ for some $\alpha \in \mathbb{C}$, then the $\mathbb{C}^{[n]}$-module

$$
\mathcal{D}_{F}:=\left\{D \in \operatorname{Der}(S) \mid D(F) \in F \cdot \mathbb{C}^{[n]}\right\}
$$

is generated by the derivation $\partial$ and the derivations $D_{i j}=D_{i j}^{F}$ for $i<j$.

From now on, the derivations $D_{F_{i j}}$, where $F=X_{1}^{m_{1}}+\cdots+X_{n}^{m_{n}}$, will be denoted by Dij. Since

$$
D_{i j}\left(X_{k}\right)=\left\{\begin{array}{clc}
-m_{j} X_{j}^{m_{j}-1} & \text { if } & k=i \\
m_{i} X_{i}^{m_{i}-1} & \text { if } & k=j \\
0 & \text { if } & k \notin\{i, j\}
\end{array}\right.
$$


so $D_{i j}(F)=0$. Then $D_{i j} \in \operatorname{Der}(S)$ induces $d_{i j}=m_{i} x_{i}^{m_{i}-1} \frac{\partial}{\partial x_{j}}-m_{j} x_{j}^{m_{j}-1} \frac{\partial}{\partial x_{i}}$ in $\operatorname{Der}\left(B_{n}^{m}\right)$. Consider the derivation

$$
E=\frac{1}{m_{1}} X_{1} \frac{\partial}{\partial X_{1}}+\cdots+\frac{1}{m_{n}} X_{n} \frac{\partial}{\partial X_{n}} .
$$

Note that $E$ satisfies $E(F)=F$. Hence, $E \in \operatorname{Der}(S)$ induces

$$
\varepsilon=\frac{1}{m_{1}} x_{1} \frac{\partial}{\partial x_{1}}+\cdots+\frac{1}{m_{n}} x_{n} \frac{\partial}{\partial x_{n}} \in \operatorname{Der}\left(B_{n}^{m}\right)
$$

As a consequence of Theorem 3 the following result is obtained:

Proposition 4 If $F=X_{1}^{m_{1}}+\cdots+X_{n}^{m_{n}}$ then $\mathcal{D}_{F}:=\{D \in \operatorname{Der}(S) \mid D(F) \in$ $F \cdot S\}$ is generated by the derivation $E$ and the derivations $D_{i j}, i<j$. In particular the $B_{n}^{m}$-module $\operatorname{Der}\left(B_{n}^{m}\right)$ is generated by the derivation $\varepsilon$ and by the derivations $d_{i j}$, for $i<j$.

Proof. Since $\left\{m_{1} X^{m_{1}-1}, \ldots, m_{n} X^{m_{n}-1}\right\}$ is a regular sequence and $E(F)=F$ the result following by Theorem 3 .

\section{Linear derivations}

This section is dedicated to the study of the linear derivations of the Fermat ring

$$
B_{n}^{m}=\mathbb{C}\left[x_{1}, \ldots, x_{n}\right],
$$

where $x_{1}^{m_{1}}+\cdots+x_{n}^{m_{n}}=0$.

A derivation $d$ of the ring $B_{n}^{m}$ is called linear if

$$
d\left(x_{i}\right)=\sum_{j=1}^{n} a_{i j} x_{j} \text { for } i=1, \ldots, n, \text { where } a_{i j} \in \mathbb{C} .
$$

The matrix $[d]=\left[a_{i j}\right]$ is called the associated matrix of the derivation $d$.

Theorem 5 Let $d \in \operatorname{Der}\left(B_{n}^{m}\right)$ be linear. If $m=\left(m_{1}, \ldots, m_{n}\right)$ with $m_{i} \geq 3$ for all $i=1, \ldots, n$, then its associated matrix $[d]$ is a diagonal matrix and has the following form

$$
\left[\begin{array}{cccc}
\frac{\alpha}{m_{1}} & & & \\
& \frac{\alpha}{m_{2}} & & \\
& & \ddots & \\
& & & \frac{\alpha}{m_{n}}
\end{array}\right] .
$$

for some $\alpha \in \mathbb{C}$. 
Proof. Let $[d]=\left[a_{i j}\right]$ be the associated matrix of $d$. Then $d\left(x_{i}\right)=\sum_{j=1}^{n} a_{i j} x_{j}$, for all $i$. Since $x_{1}^{m_{1}}+\cdots+x_{n}^{m_{n}}=0$,

$$
m_{1} x_{1}^{m_{1}-1} d\left(x_{1}\right)+\cdots+m_{n} x_{n}^{m_{i}-1} d\left(x_{n}\right)=0 .
$$

Then,

$$
0=m_{1} x_{1}^{m_{1}-1}\left(\sum_{j=1}^{n} a_{1 j} x_{j}\right)+m_{2} x_{2}^{m_{2}-1}\left(\sum_{j=1}^{n} a_{2 j} x_{j}\right)+\cdots+m_{n} x_{n}^{m_{n}-1}\left(\sum_{j=1}^{n} a_{n j} x_{j}\right)
$$

Now note that

$$
\begin{aligned}
m_{1} x_{1}^{m_{1}-1}\left(\sum_{j=1}^{n} a_{1 j} x_{j}\right) & =m_{1} a_{11}\left(x_{1}^{m_{1}}\right)+m_{1} \sum_{j \neq 1}^{n} a_{1 j} x_{j} x_{1}^{m_{1}-1} \\
& =m_{1} a_{11}\left(-x_{2}^{m_{2}}-\cdots-x_{n}^{m_{n}}\right)+m_{1} \sum_{j \neq 1}^{n} a_{1 j} x_{j} x_{1}^{m_{1}-1}
\end{aligned}
$$

and

$$
\begin{gathered}
m_{2} x_{2}^{m_{2}-1}\left(\sum_{j=1}^{n} a_{2 j} x_{j}\right)=m_{2} a_{22} x_{2}^{m_{2}}+m_{2} \sum_{j \neq 2}^{n} a_{2 j} x_{j} x_{2}^{m_{2}-1} \\
\vdots \\
m_{n} x_{n}^{m_{n}-1}\left(\sum_{j=1}^{n} a_{n j} x_{j}\right)=m_{n} a_{n n} x_{n}^{m_{n}}+m_{n} \sum_{j \neq n}^{n} a_{n j} x_{j} x_{n}^{m_{n}-1}
\end{gathered}
$$

replacing in the Equation (2.1) we obtain

$$
\begin{aligned}
& 0=\left(m_{2} a_{22}-m_{1} a_{11}\right) x_{2}^{m_{2}}+\cdots+\left(m_{n} a_{n n}-m_{1} a_{11}\right) x_{n}^{m_{n}}+m_{1} \sum_{j \neq 1}^{n} a_{1 j} x_{j} x_{1}^{m_{1}-1}+ \\
& m_{2} \sum_{j \neq 2}^{n} a_{2 j} x_{j} x_{2}^{m_{2}-1}+\cdots+m_{n} \sum_{j \neq n}^{n} a_{n j} x_{j} x_{n}^{m_{n}-1} .
\end{aligned}
$$

Observe that if $m_{i} \geq 3$, then

$$
\left\{x_{2}^{m_{1}}, \ldots, x_{n}^{m_{n}}\right\} \cup\left\{x_{j} x_{i}^{m_{i}-1} \mid 1 \leq i<j \leq n,\right\} \cup\left\{x_{j} x_{i}^{m_{i}-1} \mid 1 \leq j<i \leq n\right\}
$$

is a linearly independent set over $\mathbb{C}$. Thus, we conclude that

$$
m_{n} a_{n n}=\cdots=m_{2} a_{22}=m_{1} a_{11}=\alpha \text { and } a_{i j}=0 \text { if } i \neq j,
$$


i.e.

$$
a_{i j}=\left\{\begin{array}{ccc}
0 & \text { if } & i \neq j \\
\frac{\alpha}{m_{i}} & \text { if } & i=j
\end{array}\right.
$$

This theorem shows that for $m=\left(m_{1}, \ldots, m_{n}\right)$ and $m_{i} \geq 3$ linear derivations of $B_{2}^{m}$ are what is called diagonal derivations.

The next result characterizes linear derivations of $B_{n}^{m}$ whenever $m=(2, \ldots, 2)$. Previously, remember that a square matrix with complex elements $A$ is said to be skew-symmetric matrix if $A^{T}=-A$ (here $A^{T}$ stands, of course, for the transpose of the matrix $A$ ).

Theorem 6 Let $d \in \operatorname{Der}\left(B_{n}^{m}\right)$ be linear. If $m=(2, \ldots, 2)$, then there exist a scalar derivation $d_{\alpha}\left(\left[d_{\alpha}\right]\right.$ is a scalar matrix) and a skew-symmetric derivation $d_{s}\left(\left[d_{s}\right]\right.$ a skew-symmetric matrix) such that $d=d_{\alpha}+d_{s}$. This decomposition is unique.

Proof. Let $d \in \operatorname{Der}\left(B_{n}^{m}\right)$ be a linear derivation and $A=\left[a_{i j}\right]$ its associated matrix. Using the same arguments used in Theorem 5 we obtain

$$
0=\left(a_{22}-a_{11}\right) x_{2}^{2}+\cdots+\left(a_{n n}-a_{11}\right) x_{n}^{2}+\sum_{i<j}\left(a_{i j}+a_{j i}\right) x_{i} x_{j}
$$

Since the set $\left\{x_{2}^{2}, \ldots, x_{n}^{2}\right\} \cup\left\{x_{i} x_{j} ; 1 \leq i<j \leq n\right\}$ is linearly independent over $\mathbb{C}$, it follows that

$$
a_{11}=a_{22}=\cdots=a_{n n}=\alpha \text { and } a_{i j}=-a_{j i} \text { if } i<j,
$$

then its associated matrix $[d]$ has the following form

$$
\left[\begin{array}{cccc}
\alpha & a_{12} & \ldots & a_{1 n} \\
-a_{12} & \alpha & & a_{2 n} \\
\vdots & \vdots & \ddots & \vdots \\
-a_{1 n} & -a_{2 n} & \ldots & \alpha
\end{array}\right] .
$$

where $\alpha, a_{i j} \in \mathbb{C}$. Now define $d_{\alpha}$ by $d_{\alpha}\left(x_{i}\right)=\alpha x_{i}, i=1, \ldots, n$ and $d_{s}=d-d_{\alpha}$.

\section{$3 \quad$ Locally Nilpotent Derivations}

In this section we proof that the unique locally nilpotent derivation linear of $B_{n}^{m}$ for $m=\left(m_{1}, \ldots, m_{n}\right)$ and $m_{i} \geq 3$ is the zero derivation. Further, we show that a certain class of derivations of $\mathbb{C}\left[X_{1}, \ldots, X_{n}\right]$ do not induce nonzero locally nilpotent derivation of $B_{n}^{m}$.

Let $S=\frac{\mathbb{C}^{[n]}}{I}$ be a finitely generated $\mathbb{C}$-algebra. Consider the $\mathbb{C}^{[n]}$-submodule $\mathcal{D}_{I}=\left\{D \in \operatorname{Der}_{\mathbb{C}}\left(\mathbb{C}^{[n]}\right) \mid D(I) \subseteq I\right\}$ of the module $\operatorname{Der}_{\mathbb{C}}\left(\mathbb{C}^{[n]}\right)$. It is well known 
that the $\mathbb{C}^{[n]}$-homomorfism $\varphi: \mathcal{D}_{I} \rightarrow \operatorname{Der}_{\mathbb{C}}(S)$ given by $\varphi(D)(g+I)=D(g)+I$ induces

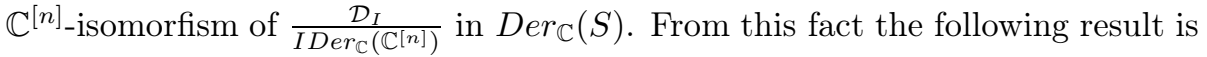
obtained.

Proposition 7 Let $d$ be a derivation of the $B_{n}^{m}$. If $d\left(x_{1}\right)=a \in \mathbb{C}$ and for each $i, 1<i \leq n, d\left(x_{i}\right) \in \mathbb{C}\left[x_{1}, \ldots, x_{i-1}\right]$, then $d$ is the zero derivation.

Proof. $\quad$ Let $F$ be the polynomial $X_{1}^{m_{1}}+\cdots+X_{n}^{m_{n}}$. We know that exists $D \in \operatorname{Der}\left(\mathbb{C}^{[n]}\right)$ such that $D(F) \in F \mathbb{C}^{[n]}$ and that $d\left(x_{i}\right)=D\left(X_{i}\right)+$ $F \mathbb{C}^{[n]}, \forall i$. Thus $D\left(X_{1}\right)-a \in F \mathbb{C}^{[n]}$, and for each $i>1$ there exists $G_{i}=$ $G_{i}\left(X_{1}, \ldots, X_{i-1}\right) \in \mathbb{C}\left[X_{1}, \ldots, X_{i-1}\right]$ such that $D\left(X_{i}\right)-G_{i} \in F \mathbb{C}^{[n]}$. Since $D(F)=\sum_{i=1}^{n} m_{i} X_{i}^{m_{i}-1} D\left(X_{i}\right) \in F \mathbb{C}^{[n]}$ and

$$
D(F)=\sum_{i=1}^{n} m_{i} X_{i}^{m_{i}-1}\left(D\left(X_{i}\right)-G_{i}\right)+\sum_{i=1}^{n} m_{i} X_{i}^{m_{i}-1} G_{i}
$$

where $G_{1}=a$, we obtain $\sum_{i=1}^{n} m_{i} X_{i}^{m_{i}-1} G_{i} \in F \mathbb{C}^{[n]}$ and then obviously $G_{i}=0$ for all $i$. Thus $d$ is the zero derivation.

Lemma 8 Let $d$ be a linear derivation of $B_{n}^{m}$ and $\left[a_{i j}\right]$ its associated matrix. Then $d$ is locally nilpotent if and only if $\left[a_{i j}\right]$ is nilpotent.

Proof. The following equality can be verified by induction over $s$.

$$
\left[\begin{array}{c}
d^{s}\left(x_{1}\right) \\
\vdots \\
d^{s}\left(x_{n}\right)
\end{array}\right]=\left[a_{i j}\right]^{s}\left[\begin{array}{c}
x_{1} \\
\vdots \\
x_{n}
\end{array}\right] .
$$

We know that $d$ is locally nilpotent if and only if there exists $r \in \mathbb{N}$ such that $d^{r}\left(x_{i}\right)=0$ for all $i$. As $\left\{x_{1}, \ldots, x_{n}\right\}$ is linearly independent over $\mathbb{C}$ by the (3.3), the result follows.

Theorem 9 If $d \in L N D\left(B_{n}^{m}\right)$ is linear and $m=\left(m_{1}, \ldots, m_{n}\right)$ wich $m_{i} \geq 3$, then $d$ is the zero derivation.

Proof. Since $d$ is locally nilpotent, $[d]$ is nilpotent (by Lemma 8) and diagonal (by Theorem 5). Thus, the matrix $[d]$ is null and $d$ is the zero derivation.

In the case $m=(2, \ldots, 2)$, linear locally nilpotent derivations of the ring $B_{n}^{m}$ were characterized as follows.

Theorem 10 [1, Theorem 1] If $d \in \operatorname{Der}\left(B_{n}^{m}\right)$ is linear and $m=(2, \ldots, 2)$, then $d \in L N D\left(B_{n}^{2}\right)$ if, and only if, its associated matrix is nilpotent and skewsymmetric. 


\section{Ring of constants}

In this section we show that the ring of constants of all nonzero linear derivations of $B_{n}^{m}$, where $m=\left(m_{1}, \ldots, m_{n}\right)$ and $m_{i} \geq 3$, is trivial, that is $\operatorname{ker}(d)=\mathbb{C}$.

During all this section we always consider $m=\left(m_{1}, \ldots, m_{n}\right)$ with $m_{i} \geq 3$.

The next result ensures the existence of Darboux elements for every nonzero linear derivation of $B_{n}^{m}$.

Proposition 11 Let $d$ be a nonzero linear derivation of $B_{n}^{m}$. If $d\left(x_{i}\right)=\frac{\alpha}{m_{i}} x_{i}$, $i=1, \ldots, n$, for some $\alpha \in \mathbb{C}^{*}$, then $f=b x_{1}^{i_{1}} \cdots x_{n}^{i_{n}}$ is a Darboux element of $d$, $d(f)=\lambda f$, and

$$
\lambda=\alpha\left(\frac{i_{1}}{m_{1}}+\frac{i_{2}}{m_{2}}+\cdots+\frac{i_{n}}{m_{n}}\right)
$$

Proof. Let $f=b x_{1}^{i_{1}} \cdots x_{n}^{i_{n}}$. Then

$$
\begin{aligned}
d(f) & =d\left(b x_{1}^{i_{1}} \cdots x_{n}^{i_{n}}\right) \\
& =b d\left(x_{1}^{i_{1}} \cdots x_{n}^{i_{n}}\right) \\
& =b \sum_{k=1}^{n} i_{k} x_{1}^{i_{1}} \cdots x_{k}^{i_{k}-1} \cdots x_{n}^{i_{n}} d\left(x_{k}\right) \\
& =b \sum_{k=1}^{n} i_{k} x_{1}^{i_{1}} \cdots x_{k}^{i_{k}-1} \cdots x_{n}^{i_{n}}\left(\frac{\alpha}{m_{k}} x_{k}\right) \\
& =\alpha b \sum_{k=1}^{n} \frac{i_{k}}{m_{k}} x_{1}^{i_{1}} \cdots x_{n}^{i_{n}} \\
& =b \alpha\left(\frac{i_{1}}{m_{1}}+\frac{i_{2}}{m_{2}}+\cdots+\frac{i_{n}}{m_{n}}\right) x_{1}^{i_{1}} \cdots x_{n}^{i_{n}} \\
& =\lambda f
\end{aligned}
$$

Corollary 12 Let $d$ be a nonzero linear derivation of $B_{n}^{m}$. If $f$ is a homogeneous element of degree $k$, then $f$ is a Darboux element of $B_{n}^{m}$ with eigenvalue $\lambda=\frac{k}{m}$.

Proof. Let $f=\sum_{i_{1}+\cdots+i_{n}=k} a_{\left(i_{1} \cdots i_{n}\right)} x_{1}^{i_{1}} \cdots x_{n}^{i_{n}}$ be a homogeneous element of degree $k$, where $0 \leq i_{n}<m$ and $a_{\left(i_{1} \cdots i_{n}\right)} \in \mathbb{C}$. 


$$
\begin{aligned}
d(f) & =d\left(\sum_{i_{1}+\cdots+i_{n}=k} a_{\left(i_{1} \cdots i_{n}\right)} x_{1}^{i_{1}} \cdots x_{n}^{i_{n}}\right) \\
& =\sum_{i_{1}+\cdots+i_{n}=k} a_{\left(i_{1} \cdots i_{n}\right)} d\left(x_{1}^{i_{1}} \cdots x_{n}^{i_{n}}\right) \\
& =\sum_{i_{1}+\cdots+i_{n}=k} a_{\left(i_{1} \cdots i_{n}\right)}\left(\frac{i_{1}}{m}+\frac{i_{2}}{m}+\cdots+\frac{i_{n}}{m}\right) x_{1}^{i_{1}} \cdots x_{n}^{i_{n}} \\
& =\sum_{i_{1}+\cdots+i_{n}=k} a_{\left(i_{1} \cdots i_{n}\right)} \frac{k}{m} x_{1}^{i_{1}} \cdots x_{n}^{i_{n}} \\
& =\frac{k}{m}\left(\sum_{i_{1}+\cdots+i_{n}=k} a_{\left(i_{1} \cdots i_{n}\right)} x_{1}^{i_{1}} \cdots x_{n}^{i_{n}}\right) \\
& =\lambda f
\end{aligned}
$$

The main result this section is:

Theorem 13 Let d be a nonzero linear derivation of $B_{n}^{m}$. Then $\operatorname{ker}(d)=\mathbb{C}$.

Proof. By Theorem $5, d\left(x_{i}\right)=\frac{\alpha}{m_{i}} x_{i}$ for $i=1, \ldots, n$ and $\alpha \in \mathbb{C}$. Since $d \neq 0$, so $\alpha \neq 0$. Let $0 \neq f \in B_{n}^{m}$ such that $d(f)=0$. Thus $f=\sum_{\left(i_{1}, \ldots, i_{n}\right) \in I} b_{\left(i_{1}, \ldots, i_{n}\right)} x_{1}^{i_{1}} \cdots x_{n}^{i_{n}}$ where $0 \neq b_{\left(i_{1}, \ldots, i_{n}\right)} \in \mathbb{C}$ for all $\left(i_{1}, \ldots, i_{n}\right) \in I$. Then

$$
\begin{aligned}
0=d(f) & =\sum b_{\left(i_{1} \cdots i_{n}\right)} d\left(x_{1}^{i_{1}} \cdots x_{n}^{i_{n}}\right) \\
& =\sum b_{\left(i_{1} \cdots i_{n}\right)} \alpha\left(\frac{i_{1}}{m_{1}}+\frac{i_{2}}{m_{2}}+\cdots+\frac{i_{n}}{m_{n}}\right) x_{1}^{i_{1}} \cdots x_{n}^{i_{n}}
\end{aligned}
$$

It follows from Lemma 1 that $b_{\left(i_{1} \cdots i_{n}\right)} \alpha\left(\frac{i_{1}}{m_{1}}+\frac{i_{2}}{m_{2}}+\cdots+\frac{i_{n}}{m_{n}}\right) \neq 0$ for all $\left(i_{1}, \ldots, i_{n}\right) \in I$, because $b_{\left(i_{1} \cdots i_{n}\right)} \alpha \neq 0$ for all $\left(i_{1}, \ldots, i_{n}\right) \in I$. So $\frac{i_{1}}{m_{1}}+\frac{i_{2}}{m_{2}}+$ $\cdots+\frac{i_{n}}{m_{n}}=0$ for all $\left(i_{1}, \ldots, i_{n}\right) \in I$. This implies that $\left(i_{1}, \ldots, i_{n}\right)=(0, \ldots, 0)$ for all $\left(i_{1}, \ldots, i_{n}\right) \in I$. Therefore $f \in \mathbb{C}$.

Theorem 14 Let $d \in \operatorname{Der}\left(B_{n}^{m}\right)$ be given by $d\left(x_{i}\right)=\frac{\alpha}{m_{i}}, i=1, \ldots, n$, for some $\alpha \in \mathbb{C}$. If

$$
f=\sum_{\left(i_{1}, \ldots, i_{n}\right) \in I} a_{\left(i_{1}, \ldots, i_{n}\right)} x_{1}^{i_{1}} \cdots x_{n}^{i_{n}}
$$

is a darboux element of $d$, this is, $d(f)=\lambda f$ for some $\lambda \in B_{n}^{m}$, then $\lambda=\alpha\left(\frac{i_{1}}{m_{1}}+\frac{i_{2}}{m_{2}}+\cdots+\frac{i_{n}}{m_{n}}\right)$ for all $\left(i_{1}, \ldots, i_{n}\right) \in I$. 
Proof. Let $f=\sum a_{\left(i_{1}, \ldots, i_{n}\right)} x_{1}^{i_{1}} \cdots x_{n}^{i_{n}}$. It follows from Theorems 13 and 5 that

$$
d(f)=\sum_{\left(i_{1}, \ldots, i_{n}\right) \in I} a_{\left(i_{1} \cdots i_{n}\right)} b_{\left(i_{1} \ldots i_{n}\right)} x_{1}^{i_{1}} \cdots x_{n}^{i_{n}}
$$

where $b_{\left(i_{1} \cdots i_{n}\right)}=\alpha\left(\frac{i_{1}}{m_{1}}+\frac{i_{2}}{m_{2}}+\cdots+\frac{i_{n}}{m_{n}}\right) \in \mathbb{C}$. Then

$$
\sum a_{\left(i_{1} \cdots i_{n}\right)} b_{\left(i_{1} \ldots i_{n}\right)} x_{1}^{i_{1}} \cdots x_{n}^{i_{n}}=d(f)=\lambda f=\sum \lambda a_{\left(i_{1} \cdots i_{n}\right)} x_{1}^{i_{1}} \cdots x_{n}^{i_{n}}
$$

So

$$
a_{\left(i_{1} \ldots i_{n}\right)} b_{\left(i_{1} \cdots i_{n}\right)}=\lambda a_{\left(i_{1} \ldots i_{n}\right)}
$$

for all $\left(i_{1}, \ldots, i_{n}\right) \in I$, by Lemma 1 . Therefore $\lambda=b_{\left(i_{1} \ldots i_{n}\right)}=\alpha\left(\frac{i_{1}}{m_{1}}+\frac{i_{2}}{m_{2}}+\right.$ $\left.\cdots+\frac{i_{n}}{m_{n}}\right)$ for all $\left(i_{1}, \ldots, i_{n}\right) \in I$.

\section{The case $m=(2, \ldots, 2)$}

In this section we focus on the Fermat rings

$$
B_{n}^{m}=\frac{\mathbb{C}\left[X_{1}, \ldots, X_{n}\right]}{\left(X_{1}^{2}+\cdots+X_{n}^{2}\right)}
$$

where $m=(2, \ldots, 2)$ and $n \geq 3$. For simplicity we denote $B_{n}^{m}$ by $B_{n}^{2}$.

We study linear derivations of $B_{n}^{2}$, their rings of constants, and we show how to construct examples of linear derivations with trivial ring of constants.

The next result will be useful for this purpose.

Proposition 15 Let $d$ be a nonzero linear derivation of $B_{n}^{m}$, with $d=d_{\alpha}+d_{s}$, where $d_{\alpha}$ is the scalar derivation and is skew-symmetric derivation. Let $d_{\alpha}$ definid by $d_{\alpha}\left(x_{i}\right)=\alpha x_{i}$ for $i=1, \ldots, n$ and $\alpha \in \mathbb{C}$. If $f \in B_{n}^{2}$ is homogeneous element of degree $k$ then $d(f)=\lambda f$ if, and only if, $d_{s}(f)=(\lambda-k \alpha) f$.

Proof. It is easy to see that $d_{\alpha}(f)=k a f$. Suppose that $d(f)=\lambda f$. Then

$$
\lambda f=d(f)=d_{\alpha}(f)+d_{s}(f)=-\alpha k f+d_{s}(f) .
$$

Hence,

$$
d_{s}(f)=(\lambda-k \alpha) f .
$$

Now suppose $d_{1}(f)=(\lambda-k a) f$. Then

$$
d(f)=d_{\alpha}(f)+d_{s}(f)=k \alpha f+(\lambda-k \alpha) f=\lambda f .
$$

Corollary 16 Let $d=d_{\alpha}+d_{s}$ be a nonzero linear derivation of $B_{n}^{2}$, where $d_{\alpha}$ is the scalar derivation and $d_{s}$ is skew-symmetric derivation. If $f \in B_{n}^{2}$ is homogeneous element of degree $k$ then $d(f)=0$ if, and only if, $d_{s}(f)=-k \alpha f$. 
Proof. Consider $\lambda=0$ in Proposition 15.

The next Theorem shows that every skew-symmetric derivation has a nontrivial ring of constants and every nonzero scalar derivation has trivial ring of constants.

Theorem 17 Let $d=d_{\alpha}+d_{s}$ be a nonzero linear derivation of $B_{n}^{2}$, where $d_{\alpha}$ is the scalar derivation and $d_{s}$ is skew-symmetric derivation. Then

1. If $d_{s}$ is zero the derivation, then $\operatorname{ker}(d)=\operatorname{ker}\left(d_{\alpha}\right)$ is trivial.

2. If $d_{\alpha}$ is the zero derivation, then $\operatorname{ker}(d)=\operatorname{ker}\left(d_{s}\right)$ is nontrivial.

Proof. 1) Observe that $d_{\alpha}(f)=k a f$ for all homogeneous element of degree $k$ and $d_{\alpha}\left(\mathcal{V}_{k}\right) \subset \mathcal{V}_{k}$.

2) It suffices to prove that there is $f \in B_{n}^{2}$ such that $d_{s}(f)=0$ and $f \notin \mathbb{C}$. Let $f$ a homogeneous element of degree 2 of $B_{n}^{2}$, then $f=X A X^{T}$ where $A=\left[a_{i j}\right]$ is a symmetric matrix and $X=\left(x_{1}, \ldots, x_{n}\right)$. Observe that for $B=\left[d_{s}\right]$ we have

$$
\begin{aligned}
d(f)=d\left(X A X^{T}\right) & =(X B) A X^{T}+X A(X B)^{T} \\
& =X B A X^{T}+X A\left(-B X^{T}\right) \\
& =X B A X^{T}-X A B X^{T} \\
& =X(B A-A B) X^{T} .
\end{aligned}
$$

If $B^{2} \neq 0$ consider the symmetric matrix $B^{2}$. It follows from the above remark that for $f=X B^{2} X^{T}$ we have $d_{s}(f)=0$ and $f \notin \mathbb{C}$, because $A=B^{2} \neq 0$. If $B^{2}=0$, then $\lambda=0$ is eigenvalue of $B$ and $B^{T}$. In this case, choose a nonzero element $f=a_{1} x_{1}+\cdots+a_{n} x_{n}$ such that the nonzero vector $\left(a_{1}, \ldots, a_{n}\right)^{T}$ is an eigenvector of $B^{T}$. So $d(f)=0$ and $f \notin \mathbb{C}$. Therefore, $\operatorname{ker}\left(d_{s}\right) \neq \mathbb{C}$.

We also show that there are linear derivations with $d_{\alpha} \neq 0, d_{s} \neq 0$, and trivial ring of constants.

Theorem 18 Let $d_{s}$ be a nonzero skew-symmetric derivation of $B_{n}^{2}$. Then exists a scalar derivation $d_{\alpha}$ of $B_{n}^{2}$ such that the derivation $d=d_{\alpha}+d_{s}$ satisfies $\operatorname{ker}(d)=\mathbb{C}$.

Proof. First note that the vector space $\mathcal{V}_{k}$ (the set of homogeneous elements of degree $k$ of $\left.B_{n}^{2}\right)$ is invariant with respect to $d_{s}$. Hence $d_{s}(f)=0$ if only if $d_{s}\left(f_{k}\right)=0$ for all homogeneous components $f_{k}$ of $f$. As a consequence of this fact we assume that $f$ is a homogeneous element of degree $k$. Let $\alpha$ be a nonzero complex number that satisfies the conditions

1. $\alpha \notin \operatorname{Spec}\left(d_{s}\right)$,

2. for all positive integer $k,-k \alpha \notin \operatorname{Spec}\left(d_{s} \mid \mathcal{V}_{k}\right)$. 
This number exists because $\mathbb{C}$ is uncountable and the set of the numbers that satisfies 1) and 2) are countable. Let $\alpha$ be a number that satisfies the conditions 1 ) and 2), then $d_{s}(f) \neq \alpha f$ and $d_{s}(f) \neq-k \alpha f$, for all $f \notin \mathbb{C}$ and for all positive integer $k$. Let $d_{\alpha}$ be a scalar derivation defined by $d_{\alpha}\left(x_{i}\right)=\alpha x_{i}, i=1, \ldots, n$.

Finally, by considering the derivation $d=d_{\alpha}+d_{s}$, we show that $\operatorname{ker}(d)=\mathbb{C}$. In order to do that, if $g \in B_{n}^{2}$ is a nonzero homogeneous element of degree $k$, then $d(g)=0$ if, and only if, $d_{s}(g)=-k \alpha g$, by Corollary 16, which implies $k=0$. Therefore, $g \in \mathbb{C}^{*}$.

We now provide and explicit example of such derivation:

Example 19 Let $d=d_{1}+d_{s}$ be the linear derivation of $B_{3}^{2}=\mathbb{C}[x, y, z]$ given by

$$
[d]=\left[d_{1}\right]+\left[d_{s}\right]=\left[\begin{array}{rrr}
1 & 0 & 0 \\
0 & 1 & -1 \\
0 & 1 & 1
\end{array}\right]=\left[\begin{array}{lll}
1 & 0 & 0 \\
0 & 1 & 0 \\
0 & 0 & 1
\end{array}\right]+\left[\begin{array}{rrr}
0 & 0 & 0 \\
0 & 0 & -1 \\
0 & 1 & 0
\end{array}\right] .
$$

We claim that $\operatorname{ker}(d)=\mathbb{C}$. To be more precise, let $\mathcal{V}_{k}$ be the set of all homogeneous elements of degree $k$ in $\mathbb{C}[y, z]$. The set

$$
S_{k}=\left\{y^{k}, y^{k-1} z, \ldots, y z^{k-1}, z^{k}\right\}
$$

is a basis for $\mathcal{V}_{k}$. The matrix

$$
\left[d \mid \mathcal{V}_{k}\right]=\left[\begin{array}{ccccccc}
k & 1 & 0 & \ldots & \ldots & 0 & 0 \\
-k & k & 2 & \cdots & \cdots & 0 & 0 \\
0 & -(k-1) & k & \ddots & & \vdots & \vdots \\
& 0 & -(k-2) & \ddots & k-2 & 0 & 0 \\
\vdots & \vdots & 0 & \ddots & k & k-1 & 0 \\
0 & 0 & \vdots & & -2 & k & k \\
0 & 0 & 0 & \ldots & 0 & -1 & k
\end{array}\right]
$$

is the matrix the linear derivation $d$ restrict to subspace $\mathcal{V}_{k}$ in the basis $S_{k}$. It is easy check that $\operatorname{Det}\left(\left[d \mid \mathcal{V}_{k}\right]\right) \neq 0$ for all $k \geq 1$, by the principle of induction. Then $d(f) \neq 0$ for all homogeneous elements of degree $k \geq 1$. Therefore, $\operatorname{ker}(d)=\mathbb{C}$.

Theorem 20 Let $d=d_{\alpha}+d_{s}$ be a nonzero linear derivation of $B_{n}^{2}$, where $d_{\alpha}$ is a scalar derivation and $d_{s}$ is a skew-symmetric derivation. If $d_{s}$ is a locally nilpotent derivation then $\operatorname{ker}(d)=\mathbb{C}$, for all nonzero scalar derivation $d_{\alpha}$.

Proof. Let $0 \neq f \in B_{n}^{2}$ such that $d(f)=0$. It suffices to show that $f \in \mathbb{C}$. We may assume that $f$ is a nonzero homogeneous element of degree $k$, because $V_{k}$ is invariant by $d$. Let $m$ be the smallest positive integer such that $g=d_{s}^{m-1}(f) \neq 0$ and $d_{s}^{m}(f)=0$, this $m$ exists because $d_{s}$ is a locally nilpotent derivation. This 
implies that $g$ is a nonzero homogeneous element of degree $k$, because $V_{k}$ is invariant by $d$. One easily verifies that $d_{\alpha} d_{s}=d_{s} d_{\alpha}$ and $d d_{s}=d_{s} d$. Note that $d(g)=d_{\alpha}(g)+d_{s}(g)=k \alpha g$, because $g$ is an homogeneous element of degree $k$ and $d_{s}^{m}(f)=0$. Now observe that

$$
d(g)=d\left(d_{s}^{m-1}(f)\right)=d_{s}^{m-1}(d(f))=d_{s}^{m-1}(0)=0 .
$$

We thus get $k \alpha g=0$. And so $k=0$. Therefore, $f \in \mathbb{C}$.

To conclude this section we construct two families of examples which illustrate this theorem.

Example 21 Let $n \geq 3$ be an odd number and $d_{s}$ a linear derivation of $B_{n}^{2}$ defined by the skew-symmetric matrix $n \times n$

$$
\left[d_{s}\right]=\left[\begin{array}{cccccc}
0 & 0 & \ldots & 0 & 0 & -1 \\
0 & 0 & \ldots & 0 & 0 & -i \\
\vdots & \vdots & \ddots & \vdots & \vdots & \vdots \\
0 & 0 & \ldots & 0 & 0 & -1 \\
0 & 0 & \ldots & 0 & 0 & -i \\
1 & i & \ldots & 1 & i & 0
\end{array}\right] .
$$

It is easy to check that $\left[d_{s}\right]^{3}=0$, which implies that $\left[d_{s}\right]$ is nilpotent. Then $d_{s}$ is a locally nilpotent linear derivation of $B_{n}^{2}$, by Theorem 10 . Now consider the linear derivation $d=d_{1}+d_{s}$, where $d_{1}\left(x_{i}\right)=x_{i}$ for $i=1, \ldots, n$. It follows from Theorem 20 that $\operatorname{ker}\left(d_{s}\right)=\mathbb{C}$.

Example 22 Let $n \geq 4$ be an even number and $\varepsilon \in \mathbb{C}$ a primitive $(n-1)$-th root of unity. Set $d_{s}$ a linear derivation of $B_{n}^{2}$ by the skew-symmetric matrix $n \times n$

$$
\left[d_{s}\right]=\left[\begin{array}{ccccccc}
0 & 0 & \ldots & 0 & \ldots & 0 & -1 \\
0 & 0 & 0 & \ldots & 0 & 0 & -\varepsilon \\
\vdots & \vdots & \ddots & \vdots & \ddots & \vdots & \vdots \\
0 & 0 & \ldots & 0 & \ldots & 0 & -\varepsilon^{k} \\
\vdots & \vdots & \ddots & \vdots & \ddots & \vdots & \vdots \\
0 & 0 & \ldots & 0 & \ldots & 0 & -\varepsilon^{n-2} \\
1 & \varepsilon & \ldots & \varepsilon^{k} & \ldots & \varepsilon^{n-2} & 0
\end{array}\right]
$$

Again, $\left[d_{s}\right]$ is nilpotent $\left(\left[d_{e}\right]^{3}=0\right)$. Thus, $d_{s}$ is a locally nilpotent derivation of $B_{n}^{2}$, by Theorem 10. Now considering the linear derivation $d=d_{1}+d_{e}$, where $d_{1}$ is the same as in the previous example, we conclude that $\operatorname{ker}\left(d_{s}\right)=\mathbb{C}$, by Theorem 20.

Remark: In the Example 19 it easy see that $\left[d_{s}\right]$ is not nilpotent and, consequently $d_{s}$ is not locally nilpotent (Theorem 10). This shows that $d_{s}$ locally nilpotent is not a necessary condition in Theorem 20 for $\operatorname{ker}\left(d_{\alpha}+d_{s}\right)=\mathbb{C}$. 


\section{References}

[1] P. Brumatti and M. Veloso, On locally nilpotent derivations of Fermat Rings, Algebra and Discrete Mathematics 16 (1), 33-41, (2013).

[2] P. Brumatti and M. Veloso, A note on Nakai's conjecture for the ring $\frac{K\left[X_{1}, \ldots, X_{n}\right]}{\left(a_{1} X_{1}^{m_{1}}+\cdots+a_{n} X_{n}^{m_{n}}\right)}$, Colloquium Mathematicum 123 (2), 277-283, (2011).

[3] D. Fiston and S. Maubach, Constructing (almost) rigid rings and a UFD having infinitely generated Derksen and Makar-Limanov invariant, Canadian Mathematical Bulletin 53 (1), 77-86, (2010).

[4] G. Freudenberg, Algebraic Theory of Locally Nilpotent Derivations, Encyclopaedia of Mathematical Sciences 136, Springer-Verlag Berlin Heidelberg, (2006).

[5] A. Nowicki, On the nonexistence of rational first integrals for systems of linear differential equations, Linear Algebra and Its Applications 235, 107120, (1996).

[6] J. M. Ollagnie and A. Nowicki, Derivations of polynomial algebras without Darboux polynomials, Journal of Pure and Applied Algebra 212, 1626-1631, (2008).

[7] J. Zielinski, Rational Constants of Generic LV Derivations and of Monomial Derivations, Bulletin of the Polish Academy of Sciences. Mathematics 61, Issue: 3, 201-208, (2013). 